\title{
Pricing Asian Options in a Semimartingale Model*
}

\author{
Jan Večeř \\ Columbia University, Department of Statistics, New York, NY 10027, USA \\ Kyoto University, Kyoto Institute of Economic Research, Financial Engineering Center, Kyoto, Japan \\ Mingxin Xu \\ Carnegie Mellon University, Department of Mathematical Sciences, Pittsburgh, PA 15213, USA
}

First version: September 10, 2001

This version: April 10, 2002

\begin{abstract}
In this article we study arithmetic Asian options when the underlying stock is driven by special semimartingale processes. We show that the inherently path dependent problem of pricing Asian options can be transformed into a problem without path dependency in the payoff function. We also show that the price satisfies a simpler integro-differential equation in the case the stock price is driven by a process with independent increments, Lévy process being a special case.
\end{abstract}

Key words: Asian options, Special semimartingales, Lévy processes, Integro-differential equations.

\section{Introduction}

Asian options are securities with payoff which depends on the average of the underlying stock price $S$ over a certain time interval. If we denote by $\lambda$ the averaging factor of the option, we can write the general Asian option payoff as

$$
\left(\xi \cdot\left(\int_{0}^{T} S_{t} d \lambda(t)-K_{1} S_{T}-K_{2}\right)\right)^{+} .
$$

When $K_{1}=0$, we have fixed strike option; when $K_{2}=0$, we have floating strike option. The constant $\xi= \pm 1$ determines whether the option is call or put. The averaging factor $\lambda$ has finite variation and is typically taken to be

$$
\lambda(t)=\frac{t}{T}
$$

for the case of continuously sampled Asian options, or

$$
\lambda(t)=\frac{1}{n} \cdot\left\lfloor\frac{n t}{T}\right\rfloor
$$

for the case of discretely sampled Asian options. Other averaging is also possible (exponential, etc.), but less frequently used in practice. Notice that European type options are just a special case of Asian option for the following choice of parameters: $\lambda(t)=1_{\{T\}}(t)$ and $K_{1}=0$.

\footnotetext{
*Acknowledgements: We would like to thank Steven Shreve whose careful reading has significantly improved the manuscript. We have also received valuable suggestions from David Heath, Julien Hugonnier, Dmitry Kramkov, Yoshio Miyahara and Isaac Sonin. Takeaki Kariya and Financial Engineering Center of Kyoto University provided us with creative research environment, in which we have initiated this project. The work of Mingxin Xu was supported by the National Science Foundation under grant DMS-0103814.
} 
There has been a growing concern in the literature on the lognormality assumption of the underlying stock price, and a number of alternative approaches have been suggested. One of the most studied situations is the case when the stock is driven by a particular Lévy process. Carr, Geman, Madan and Yor (2000) have recently suggested the so-called CGMY model for the stock price, which shows a good match with empirical data. Another alternative approach, namely the general hyperbolic model, is discussed in Eberlein and Prause (1998).

The problem of pricing Asian options is already complicated when the underlying stock is a geometric Brownian motion. Most of the literature we know studies only this type of model. A number of approximations that produce closed form expressions have appeared, most recently in Thompson (1999), who provides tight analytical bounds for the Asian option price. Geman and Yor (1993) computed the Laplace transform of the Asian option price, but numerical inversion remains problematic for low volatility and/or short maturity cases.

Rogers and Shi (1995) have formulated a one-dimensional PDE that can model both floating and fixed strike continuous average Asian options. However this one-dimensional PDE is difficult to solve numerically since the diffusion term is very small for values of interest on the finite difference grid. Andreasen (1998) has extended this approach for pricing discretely sampled Asian option.

Monte Carlo methods seem to work well, but sampling the entire path of the underlying asset greatly reduces competitiveness of this approach, even with the help of variation reduction techniques (Fu, Madan, Wang (1998/99)).

In the recent paper of Večer (2001), it was shown that one can reformulate the problem of pricing Asian options in a way which removes the inherent path dependency of the contract. This paper applies the techniques developed in Shreve and Večeř (2000) for pricing options on a traded account. In the case when the underlying stock is a geometric Brownian motion, one can obtain a simple one-dimensional partial differential equation for the price which is easy to solve numerically. A similar formulation of the pricing partial differential equation appears in the independent work of Hoogland and Neumann (2001).

We show in this article that the approach of removing the path dependency in the formulation of the Asian option pricing problem can be generalized to the case when the underlying asset is driven by a special semimartingale process. We also show that the price satisfies an integro-differential equation in the case the stock price is driven by a process with independent increments, Lévy processes being a special case.

\section{Pricing Formula for Asian Options}

Let $H$ be a special semimartingale on the stochastic basis $\left(\Omega, \mathcal{F}, \mathbf{F}=\left(\mathcal{F}_{t}\right)_{t \in \mathbb{R}_{+}}, \mathbb{P}\right)$ that satisfies the usual conditions, with values in $\mathbb{R}$ and $H_{0}=0$. Suppose the stock price has the following dynamics:

$$
d S_{t}=S_{t-} d H_{t},
$$

We will from now on assume $\mathbb{P}$ to be a risk-neutral measure and the interest rate to be a constant $r$. In particular, we assume that $e^{-r t} S_{t}$ is a martingale under $\mathbb{P}^{\dagger}$.

Following the notation in Jacod and Shiryaev (1987), $H$ has the canonical decomposition:

$$
H_{t}=r t+H_{t}^{c}+\int_{0}^{t} \int_{-\infty}^{\infty} x(\mu(d s, d x)-\nu(d s, d x)),
$$

where $H_{t}^{c}$ is the continuous martingale part, $\mu(d t, d x)$ is the random measure associated with the jumps of $H$ and $\nu(d t, d x)$ is the compensator. According to II.2.9 and II.2.29 in Jacod and Shiryaev (1987), we can always choose a good version of $\nu$, i.e., $\nu(\{t\}, \mathbb{R}) \leq 1, \nu\left(\mathbb{R}_{+},\{0\}\right)=0$ and $\int_{0}^{t} \int_{-\infty}^{\infty}\left(|x|^{2} \wedge|x|\right) \nu(d t, d x)$ is a process with locally integrable variation. To ensure that $S$ is a positive process, we make the following assumption:

\footnotetext{
${ }^{\dagger}$ Here we will not discuss in detail how to choose this equivalent martingale measure for pricing purpose. Interested readers are referred to Föllmer and Schweizer (1991) and Miyahara (2001) for the Föllmer-Schweizer minimal measure, or equivalently, minimal entropy martingale measure; Elliott, Hunter, Kopp and Madan (1995) for the equivalent martingale measure resulting from multiplicative decomposition; Gerber and Shiu (1994) for Esscher transform. A nice presentation of these methods for geometric Lévy processes can be found in Chan (1999).
} 
Assumption $2.1 \mu([0, t],(-\infty,-1])=0$ for all $t \geq 0$.

The Doléans-Dade formula gives

$$
S_{t}=S_{0} \mathcal{E}(H)=S_{0} \exp \left(H_{t}-\frac{1}{2}\left\langle H^{c}\right\rangle_{t}\right) \prod_{0<s \leq t}\left(1+\Delta H_{s}\right) e^{-\Delta H_{s}} .
$$

We will define a new measure $\mathbb{Q}$ by

$$
\left.\frac{d \mathbb{Q}}{d \mathbb{P}}\right|_{t}=\frac{S_{t}}{S_{0} e^{r t}}
$$

and a process $Z_{t}=\frac{X_{t}}{S_{t}}$, where $X_{t}$ is the self-financing portfolio

$$
d X_{t}=q_{t-} d S_{t}+r\left(X_{t-}-q_{t-} S_{t-}\right) d t,
$$

where the predictable process $q_{t}$ represents the shares invested in stock. An equivalent formulation with discounted value about the portfolio is

$$
d\left(e^{-r t} X_{t}\right)=q_{t-} d\left(e^{-r t} S_{t}\right)
$$

In order to reformulate the pricing problem and remove the path dependency, we can use the following procedure of replicating the Asian Forward payoff. Without loss of generality, we assume $\xi=1$.

Proposition 2.2 (Replication of the Asian Forward Contract) Suppose that we have a self-financing portfolio $X$ as in (2.6) where $S_{t}$ is a semimartingale. If we set the shares invested in the stock to be

$$
q_{t}=e^{-r T} \int_{t}^{T} e^{r s} d \lambda(s)
$$

where $\lambda(t)$ is a deterministic function with finite variation, and start with the initial wealth

$$
X_{0}=q_{0} S_{0}-e^{-r T} K_{2},
$$

then we will have

$$
X_{T}=\int_{0}^{T} S_{t} d \lambda(t)-K_{2}
$$

Proof. For notational purpose, let $B_{t}=e^{-r t} S_{t}$. By the definition of quadratic variation and (2.6),

$$
e^{-r T} X_{T}-X_{0}=\int_{0}^{T} q_{t-} d B_{t}=q_{T} B_{T}-q_{0} S_{0}-\int_{0}^{T} B_{t-} d q_{t}-[q, B]_{T} .
$$

Since $q_{t}$ is of finite variation,

$$
\int_{0}^{T} B_{t-} d q_{t}+[q, B]_{t}=\int_{0}^{T} B_{t-} d q_{t}+\sum_{0<u \leq t} \Delta q_{u} \Delta B_{u}=\int_{0}^{T} B_{t} d q_{t}=\int_{0}^{T} e^{-r t} S_{t} d q_{t} .
$$

Given the formula (2.7) for $q_{t}$ (note that $q_{T}=0$ ), and the formula (2.8) for $X_{0},(2.10)$ simplifies to

$$
\begin{aligned}
X_{T} & =e^{r T} X_{0}-e^{r T} q_{0} S_{0}-\int_{0}^{T} e^{r(T-t)} S_{t} d q_{t} \\
& =\int_{0}^{T} S_{t} d \lambda(t)-K_{2} .
\end{aligned}
$$


Remark 2.3 Proposition 2.2 works for general semimargales $S_{t}$. The proof uses neither the martingale measure assumption nor the specific forms (2.1) and (2.2). It also works for general deterministic function $\lambda(t)$ with finite variation.

Theorem 2.4 (Pricing Formula) Let $V^{\lambda}\left(0, S_{0}, K_{1}, K_{2}\right)$, the price of the Asian option with the payoff (1.1) when $\xi=1$, be defined as

$$
V^{\lambda}\left(0, S_{0}, K_{1}, K_{2}\right) \triangleq E^{\mathbb{P}}\left[e^{-r T}\left(\int_{0}^{T} S_{t} d \lambda(t)-K_{1} S_{T}-K_{2}\right)^{+}\right] .
$$

Then we have the following relationship

$$
V^{\lambda}\left(0, S_{0}, K_{1}, K_{2}\right)=S_{0} \cdot \mathbb{E}^{\mathbb{Q}}\left[\left(Z_{T}-K_{1}\right)^{+}\right]
$$

where $\mathbb{Q}$ is defined by (2.4), $X_{t}$ is the self-financing portfolio (2.5) with the initial condition $X_{0}$ and trading strategy $q_{t}$ defined in (2.8) and (2.7), and $Z_{t}=\frac{X_{t}}{S_{t}}$.

Proof. An easy consequence of proposition 2.2 is

$$
\begin{aligned}
V^{\lambda}\left(0, S_{0}, K_{1}, K_{2}\right) & =e^{-r T} \cdot \mathbb{E}^{\mathbb{P}}\left[\left(\int_{0}^{T} S_{t} d \lambda(t)-K_{1} S_{T}-K_{2}\right)^{+}\right] \\
& =e^{-r T} \cdot \mathbb{E}^{\mathbb{P}}\left[\left(X_{T}-K_{1} S_{T}\right)^{+}\right] \\
& =e^{-r T} \cdot \mathbb{E}^{\mathbb{Q}}\left[\left(X_{T}-K_{1} S_{T}\right)^{+} \frac{S_{0} e^{r T}}{S_{T}}\right] \\
& =S_{0} \cdot \mathbb{E}^{\mathbb{Q}}\left[\left(Z_{T}-K_{1}\right)^{+}\right] .
\end{aligned}
$$

Remark 2.5 Theorem 2.4 works under the assumption that $e^{-r t} S_{t}$ is a strictly positive martingale under $\mathbb{P}$. The proof does not require the specific forms (2.1) and (2.2). It also works for general deterministic function $\lambda(t)$ with finite variation.

\section{Integro-Differential Equation}

For our next analysis, we need the following result:

Lemma 3.1 $Z_{t}=\frac{X_{t}}{S_{t}}$ is a local martingale under $\mathbb{Q}$.

Proof. Recall that $\mathbb{P}$ is a risk-neutral measure. (2.6) and the fact that $q_{t}$ is deterministic ensure that $e^{-r t} X_{t}$ is a martingale. For $0 \leq u \leq t$,

$$
\begin{aligned}
E^{\mathbb{Q}}\left[Z_{t} \mid \mathcal{F}_{u}\right] & =\frac{S_{0} e^{r u}}{S_{u}} E^{\mathbb{P}}\left[\frac{S_{t} Z_{t}}{S_{0} e^{r t}} \mid \mathcal{F}_{u}\right] \\
& =\frac{e^{r u}}{S_{u}} E^{\mathbb{P}}\left[e^{-r t} X_{t} \mid \mathcal{F}_{u}\right] \\
& =\frac{e^{r u}}{S_{u}} e^{-r u} X_{u}=Z_{u} .
\end{aligned}
$$


Remark 3.2 Lemma 3.1 holds in the same generality as Theorem 2.4.

If $H$ is a PII (process with independent increments) with decomposition (2.2), then we can find a deterministic function $c_{t}$, a deterministic measure-valued function $K_{t}$ and a deterministic increasing function $A_{t}$ such that

$$
\left\{\begin{array}{l}
d\left\langle H^{c}\right\rangle_{t}(\omega)=c_{t} d A_{t}(\omega) \\
\nu(d t, d x)=K_{t}(d x) d A_{t}(\omega) .
\end{array}\right.
$$

Further if $H$ is a Lévy process, then we can take $A_{t}=t, c$ to be a constant, and $K(x)$ (the Lévy measure) to be independent of $t$, to integrate $|x|^{2} \wedge|x|$, and to satisfy $K(\{0\})=0$.

Theorem 3.3 (Integro-differential equation for Asian options) Suppose that $H$ is PII with canonical decomposition (2.2). The value of the Asian option is a function of $t$ and $Z_{t}$, denoted by $v\left(t, Z_{t}\right)$, such that $V^{\lambda}\left(0, S_{0}, K_{1}, K_{2}\right)=S_{0} v\left(0, Z_{0}\right)$. Assume $v_{t}, v_{z}$ and $v_{z z}$ exist and are continuous. Then the following integrodifferential equation holds:

$$
\begin{aligned}
& \int_{0}^{t} {\left[v_{s}\left(s, Z_{s-}\right) d s+\frac{1}{2} v_{z z}\left(s, Z_{s-}\right)\left(q_{s-}-Z_{s-}\right)^{2} d\left\langle H^{c}\right\rangle_{s}\right.} \\
&\left.\quad+\int_{-\infty}^{\infty}\left\{v\left(s, Z_{s-}+\left(q_{s-}-Z_{s-}\right) \frac{x}{1+x}\right)-v\left(s, Z_{s-}\right)-v_{z}\left(s, Z_{s-}\right)\left(q_{s-}-Z_{s-}\right) \frac{x}{1+x}\right\} \nu(d s, d x)\right]=0
\end{aligned}
$$

for $0 \leq t \leq T$.

Proof. Apply Ito's formula to get

$$
\begin{aligned}
\frac{X_{T}}{S_{T}}= & \frac{X_{0}}{S_{0}}+\int_{0}^{T} \frac{1}{S_{t-}} d X_{t}-\int_{0}^{T} \frac{X_{t-}}{S_{t-}^{2}} d S_{t}-\int_{0}^{T} \frac{1}{S_{t-}^{2}} d\left\langle X^{c}, S^{c}\right\rangle_{t} \\
& +\int_{0}^{T} \frac{X_{t-}}{S_{t-}^{3}} d\left\langle S^{c}\right\rangle_{t}+\sum_{0<t \leq T}\left(\frac{X_{t}}{S_{t}}-\frac{X_{t-}}{S_{t-}}-\frac{1}{S_{t-}} \Delta X_{t}+\frac{X_{t-}}{S_{t-}^{2}} \Delta S_{t}\right) \\
= & \frac{X_{0}}{S_{0}}+\int_{0}^{T} \frac{1}{S_{t-}}\left(q_{t-} S_{t-} d H_{t}+r\left(X_{t-}-q_{t-} S_{t-}\right) d t\right) \\
& -\int_{0}^{T} \frac{X_{t-}}{S_{t-}} d H_{t}-\int_{0}^{T} q_{t-} d\left\langle H^{c}\right\rangle_{t}+\int_{0}^{T} \frac{X_{t-}}{S_{t-}} d\left\langle H^{c}\right\rangle_{t} \\
& +\sum_{0<t \leq T}\left(\frac{X_{t}}{S_{t}}-\frac{X_{t-}}{S_{t-}}-\frac{1}{S_{t-}} \Delta X_{t}+\frac{X_{t-}}{S_{t-}^{2}} \Delta S_{t}\right) .
\end{aligned}
$$

Note that

$$
\begin{gathered}
\Delta S_{t}=S_{t-} \Delta H_{t}, \quad \Delta X_{t}=q_{t-} S_{t-} \Delta H_{t} \\
\frac{X_{t}}{S_{t}}-\frac{X_{t-}}{S_{t-}}=\left(q_{t-}-\frac{X_{t-}}{S_{t-}}\right)\left(1-\frac{1}{1+\Delta H_{t}}\right)=\left(q_{t-}-\frac{X_{t-}}{S_{t-}}\right)\left(\frac{\Delta H_{t}}{1+\Delta H_{t}}\right) .
\end{gathered}
$$

We can write

$$
\begin{aligned}
d\left(\frac{X_{t}}{S_{t}}\right) & =\left(q_{t-}-\frac{X_{t-}}{S_{t-}}\right)\left(d H_{t}-r d t-d\left\langle H^{c}\right\rangle_{t}-\Delta H_{t}\right)+\frac{X_{t}}{S_{t}}-\frac{X_{t-}}{S_{t-}} \\
& =\left(q_{t-}-\frac{X_{t-}}{S_{t-}}\right)\left(d H_{t}-r d t-d\left\langle H^{c}\right\rangle_{t}-\Delta H_{t}+\frac{\Delta H_{t}}{1+\Delta H_{t}}\right) \\
& =\left(q_{t-}-\frac{X_{t-}}{S_{t-}}\right)\left(d H_{t}^{c}-d\left\langle H^{c}\right\rangle_{t}+\int_{-\infty}^{\infty} x(\mu(d t, d x)-\nu(d t, d x))-\int_{-\infty}^{\infty} \frac{x^{2}}{1+x} \mu(d t, d x)\right)
\end{aligned}
$$


or

$$
d Z_{t}=\left(q_{t-}-Z_{t-}\right)\left(d H_{t}^{c}-d\left\langle H^{c}\right\rangle_{t}+\int_{-\infty}^{\infty} x(\mu(d t, d x)-\nu(d t, d x))-\int_{-\infty}^{\infty} \frac{x^{2}}{1+x} \mu(d t, d x)\right)
$$

Observe that $Z_{t}$ is a Markovian process under $\mathbb{Q}$. Theorem 2.4 and the Markovian property give us the value process

$$
v\left(t, Z_{t}\right)=\mathbb{E}^{\mathbb{Q}}\left[\left(Z_{T}-K_{1}\right)^{+} \mid \mathcal{F}_{t}\right]
$$

which is a martingale by definition.

Note $d\left\langle Z^{c}\right\rangle_{t}=\left(q_{t-}-Z_{t-}\right)^{2} d\left\langle H^{c}\right\rangle_{t}$ and thus

$$
\begin{aligned}
& d v\left(t, Z_{t}\right)=v_{t}\left(t, Z_{t-}\right) d t+v_{z}\left(t, Z_{t-}\right) d Z+\frac{1}{2} v_{z z}\left(t, Z_{t-}\right) d\left\langle Z^{c}\right\rangle_{t} \\
& +v\left(t, Z_{t}\right)-v\left(t, Z_{t-}\right)-v_{z}\left(t, Z_{t-}\right) \Delta Z_{t} \\
& =v_{t}\left(t, Z_{t-}\right) d t+v_{z}\left(t, Z_{t-}\right) d Z+\frac{1}{2} v_{z z}\left(t, Z_{t-}\right)\left(q_{t-}-Z_{t-}\right)^{2} d\left\langle H^{c}\right\rangle_{t} \\
& +v\left(t, Z_{t-}+\left(q_{t-}-Z_{t-}\right) \frac{\Delta H}{1+\Delta H}\right)-v\left(t, Z_{t-}\right)-v_{z}\left(t, Z_{t-}\right)\left(q_{t-}-Z_{t-}\right) \frac{\Delta H}{1+\Delta H} \\
& =\text { Local Martingale }+v_{t}\left(t, Z_{t-}\right) d t+\frac{1}{2} v_{z z}\left(t, Z_{t-}\right)\left(q_{t-}-Z_{t-}\right)^{2} d\left\langle H^{c}\right\rangle_{t} \\
& +\int_{-\infty}^{\infty}\left\{v\left(t, Z_{t-}+\left(q_{t-}-Z_{t-}\right) \frac{x}{1+x}\right)\right. \\
& \left.-v\left(t, Z_{t-}\right)-v_{z}\left(t, Z_{t-}\right)\left(q_{t-}-Z_{t-}\right) \frac{x}{1+x}\right\} \nu(d t, d x) .
\end{aligned}
$$

The fact that a predictable local martingale with finite variation starting at zero is zero concludes the proof. $\diamond$

Corollary 3.4 In the case when $H$ is a Lévy process, the integro-differential equation simplifies to

$$
\begin{aligned}
v_{t}(t, z)+\frac{c}{2} v_{z z}(t, z)\left(q_{t-}\right. & -z)^{2} \\
& +\int_{-\infty}^{\infty}\left\{v\left(t, z+\left(q_{t-}-z\right) \frac{x}{1+x}\right)-v(t, z)-v_{z}(t, z)\left(q_{t-}-z\right) \frac{x}{1+x}\right\} K(d x)=0
\end{aligned}
$$

for $0 \leq t \leq T$ and $z \in \mathbb{R}$

Proof. The canonical decomposition of $H$ is

$$
H_{t}=r t+\int_{0}^{t} \sqrt{c} d W_{s}+\int_{0}^{t} \int_{-\infty}^{\infty} x(\mu(d s, d x)-K(d x) d t)
$$

where $W_{t}$ is a standard Brownian Motion. Applying theorem 3.3, we get

$$
\begin{aligned}
v_{t}\left(t, Z_{t-}\right) & +\frac{c}{2} v_{z z}\left(t, Z_{t-}\right)\left(q_{t-}-Z_{t-}\right)^{2} \\
& +\int_{-\infty}^{\infty}\left\{v\left(t, Z_{t-}+\left(q_{t-}-Z_{t-}\right) \frac{x}{1+x}\right)-v\left(t, Z_{t-}\right)-v_{z}\left(t, Z_{t-}\right)\left(q_{t-}-Z_{t-}\right) \frac{x}{1+x}\right\} K(d x)=0
\end{aligned}
$$

Since the support for $Z_{t-}$ is $\mathbb{R}$, we get the above equation.

\section{Applications to Different Lévy Models}

\section{Geometric Brownian Motion with Poisson Jump}


Let us start with a model similar to the one suggested in Andreasen (1998). Suppose that the stock price process evolves as

$$
d S_{t}=S_{t-} d H_{t}=S_{t-}\left(r d t+\sigma d W_{t}+\left(e^{\phi_{t}}-1\right) d M_{t}\right),
$$

where $W_{t}$ is a standard Brownian motion, and $M_{t}$ is a compensated Poisson process, i.e., $M_{t}=N_{t}-\lambda t$. Let $\phi_{t}$ be a Gaussian process with independent increments, and be independent of both $W_{t}$ and $N_{t}$, such that $\mathbb{E}\left[\phi_{t}\right]=\mu$ and $\operatorname{Var}\left[\phi_{t}\right]=\gamma^{2}$. Assume that $\gamma>0, \sigma>0, \mu$ are constants. In this case,

$$
\langle H\rangle_{t}=\sigma^{2} t, \quad K(x)=\frac{\lambda}{\sqrt{2 \pi} \gamma} \cdot \exp \left\{-\frac{(\ln (x+1)-\mu)^{2}}{\gamma^{2}}\right\},
$$

and $v(t, z)$ satisfies (3.4). If $\gamma=0$, then the jump size reduces to a constant $e^{\mu}-1$, i.e., $K(x)=\lambda \delta\left(\left\{e^{\mu}-1\right\}\right)$ and (3.4) simplifies to:

$$
\begin{aligned}
v_{t}(t, z)+\frac{\sigma^{2}}{2} v_{z z}(t, z)\left(q_{t-}-z\right)^{2} & \\
+ & {\left[v\left(t, z+\left(q_{t-}-z\right) \frac{\phi}{1+\phi}\right)-v(t, z)-\left(q_{t-}-z\right) v_{z}(t, z)\right] \frac{\phi}{1+\phi} \lambda=0, }
\end{aligned}
$$

for $0 \leq t \leq T$. In the geometric Brownian model, $d S_{t}=S_{t-} d H_{t}=S_{t-}\left(r d t+\sigma d W_{t}\right), \phi=0$, and we simply have

$$
v_{t}(t, z)+\frac{\sigma^{2}}{2}\left(q_{t}-z\right)^{2} v_{z z}(t, z)=0
$$

as shown in Večeř (2001).

\section{Pure Jump Processes Models: CGMY and General Hyperbolic}

In our model (2.1),

$$
d S_{t}=S_{t-} d H_{t},
$$

the stock price is a stochastic exponential of $H$. Another usual approach in the literature is to let the stock price to be a geometric exponential of the underlying:

$$
S_{t}=S_{0} e^{\widehat{H}_{t}}
$$

Applying Ito's lemma and rewriting (2.3):

$$
\begin{aligned}
d S_{t} & =e^{\widehat{H}_{t-}}\left(d \widehat{H}_{t}+\frac{1}{2} d\left\langle\widehat{H}^{c}\right\rangle_{t}+e^{\Delta \widehat{H}_{t}}-1-\Delta \widehat{H}_{t}\right) \\
S_{t} & =S_{0} \exp \left\{H_{t}-\frac{1}{2}\left\langle H^{c}\right\rangle_{t}+\sum_{0<s \leq t}\left(\ln \left(1+\Delta H_{s}\right)-\Delta H_{s}\right)\right\} .
\end{aligned}
$$

We can easily find the relationship between $H$ and $\widehat{H}$ :

$$
\begin{aligned}
H_{t} & =\widehat{H}_{t}+\frac{1}{2}\left\langle\widehat{H}^{c}\right\rangle_{t}+\sum_{0<s \leq t}\left(e^{\Delta \widehat{H}_{s}}-1-\Delta \widehat{H}_{s}\right) \\
\widehat{H}_{t} & =H_{t}-\frac{1}{2}\left\langle H^{c}\right\rangle_{t}+\sum_{0<s \leq t}\left(\ln \left(1+\Delta H_{s}\right)-\Delta H_{s}\right) .
\end{aligned}
$$

Therefore the two ways of modelling are equivalent with Assumption 2.1. If we are given the compensator $\widehat{\mu}(d t, d x)$ for the model (4.4), then the IDE in corollary (3.4) becomes

$$
\begin{aligned}
\widehat{v}_{t}(t, z)+\frac{c}{2} \widehat{v}_{z z}(t, z)\left(q_{t-}-z\right)^{2}+ & \\
\int_{-\infty}^{\infty}\left\{\widehat{v}\left(t, z+\left(q_{t-}-z\right) \frac{e^{\widehat{x}}-1}{e^{\widehat{x}}}\right)-\widehat{v}(t, z)-\widehat{v}_{z}(t, z)\left(q_{t-}-z\right) \frac{e^{\widehat{x}}-1}{e^{\widehat{x}}}\right\} \widehat{K}(d \widehat{x}) & =0
\end{aligned}
$$


because $\Delta H_{t}=e^{\Delta \widehat{H}_{t}}-1$.

We mention here two geometric exponential models with pure jump processes. One is CGMY in Carr, Geman, Madan, Yor (2000) with Lévy measure:

$$
\widehat{k}_{C G M Y}= \begin{cases}C \frac{\exp (-G|\widehat{x}|)}{\left.|\widehat{x}|\right|^{1+Y}}, & \text { for } \widehat{x}<0 \\ C \frac{\exp (-M|\widehat{x}|)}{|\widehat{x}|^{1+Y}}, & \text { for } \widehat{x}>0 .\end{cases}
$$

The other is the General Hyperbolic Model in Eberlein and Prause (1998) with Lévy measure:

$$
\widehat{k}_{E P}= \begin{cases}\frac{e^{\beta \widehat{x}}}{|\widehat{x}|}\left(\int_{0}^{\infty} \frac{\exp -\sqrt{2 y+\alpha^{2}}|\widehat{x}|}{\pi^{2} y\left(J_{\lambda}^{2}(\delta \sqrt{2 y})+Y_{\lambda}^{2}(\delta \sqrt{2 y})\right)} d y+\lambda e^{-\alpha|\widehat{x}|}\right), & \text { if } \lambda \geq 0 ; \\ \frac{e^{\beta \widehat{x}}}{|\widehat{x}|}\left(\int_{0}^{\infty} \frac{\exp -\sqrt{2 y+\alpha^{2}}|\widehat{x}|}{\pi^{2} y\left(J_{-\lambda}^{2}(\delta \sqrt{2 y})+Y_{-\lambda}^{2}(\delta \sqrt{2 y})\right)} d y\right), & \text { if } \lambda<0 ;\end{cases}
$$

where $J_{\lambda}$ and $Y_{\lambda}$ are the Bessel functions of the first and second kind respectively. In both models the value of the Asian option satisfy:

$$
\widehat{v}_{t}(t, z)+\int_{-\infty}^{\infty}\left\{\widehat{v}\left(t, z+\left(q_{t-}-z\right) \frac{e^{\widehat{x}}-1}{e^{\widehat{x}}}\right)-\widehat{v}(t, z)-\widehat{v}_{z}(t, z)\left(q_{t-}-z\right) \frac{e^{\widehat{x}}-1}{e^{\widehat{x}}}\right\} \widehat{K}(d \widehat{x})=0,
$$

for $0 \leq t \leq T$.

\section{Conclusion}

We have shown in this paper that we can remove the path dependency in the payoff function of all kinds of Asian options regardless of the dynamics of the underlying asset. This reformulation of the problem gives us an integro-differential equation for the price of the option when the stock is driven by an exponential Lévy process. This equation simplifies even more if we assume a particular stock price model, such as Geometric Brownian Motion with Poisson Jump model, the Carr, Geman, Madan, Yor model, or a general hyperbolic model. In the case of Black-Sholes model, we obtain a one-dimensional PDE which is simple and robust to implement.

\section{References}

[1] Andreasen, J. (1998): "The pricing of discretely sampled Asian and lookback options: a change of numeraire approach", The Journal of Computational Finance Vol. 2, No. 1, 5-30.

[2] Carr, P., H. Geman, D. Madan, M. Yor (2000): "The fine structure of asset returns: An empirical investigation", Working paper.

[3] Chan, T. (1999): "Pricing contingent claims on stocks driven by Lévy processes", The Annals of Applied Probability, Vol. 9, No. 2, 504-528.

[4] Curran, M. (1992): "Beyond average intelligence", Risk 5/1992, page 60.

[5] Eberlein, E., K. Prause (1998): "The generalized hyperbolic model: Financial derivatives and risk measures.", FDM-Preprint, 56.

[6] Elliott, R. J., W. C. Hunter, P. E. Kopp, D. Madan (1995): "Pricing via multiplicative price decomposition", Journal of Financial Engineering, 4, 247-262.

[7] Föllmer, H., M. Schweizer (1991): "Hedging of contingent claims under incomplete information", Applied Stochastic Analysis (M. H. A. Davis and R. J. Elliott, eds.), Gordon and Breach, New York, 389-414. 
[8] Fu, M., D. Madan, T. Wang (1998/99): "Pricing continuous Asian options: a comparison of Monte Carlo and Laplace transform inversion methods", The Journal of Computational Finance, Vol. 2, No. 2.

[9] Geman, H., M. Yor (1993): "Bessel processes, Asian option, and perpetuities", Mathematical Finance, 3, 349-375.

[10] Gerber, H. U., E. S. W. Shiu (1994): "Option pricing by Esscher transforms (with discussion)", Trans. Soc. Actuaries, 46, 99-191.

[11] Hoogland, J., D. Neumann (2001): "Local Scale Invariance and Contingent Claim Pricing", International Journal of Theoretical and Applied Finance, 4/1, 1-21.

[12] Hoogland, J., D. Neumann (2000): "Asians and cash dividends: Exploiting symmetries in pricing theory", Working paper.

[13] Jacod, J., A. N. Shiryaev (1987): Limit Theorems for Stochastic Processes, Springer-Verlag.

[14] Miyahara, Y. (2001): " [Geometric Lévy Process \& MEMM] Pricing Model and Related Estimation Problems", Asia-Pacific Financial Markets, Vol. 8, No. 1, 45-60.

[15] Rogers, C., Z. Shi (1995): "The value of an Asian option", Journal of Applied Probability, 32, 1077-1088.

[16] Shreve, S., J. VeČĚr (2000): "Options on a traded account: Vacation calls, vacation puts and passport options", Finance and Stochastics, 255-274.

[17] Thompson, G., (1999): "Fast narrow bounds on the value of Asian options", Working paper.

[18] VeČĚ̌, J. (2001): "A new PDE approach for pricing arithmetic average Asian options", The Journal of Computational Finance, Vol. 4, No. 4, 105-113.

[19] VeČĚr, J. (2001): "New Pricing of Asian Options", Risk, submitted. 